\title{
Transient Electromagnetic Method in the Keritis basin (Crete, Greece): Evidence of hierarchy in a complex geological structure in view of Tsallis distribution
}

\author{
Filippos Vallianatos ${ }^{1, *}$ \\ ${ }^{1}$ Laboratory of Geophysics and Seismology, UNESCO Chair on Solid Earth Physics and Geohazards Risk Reduction, \\ Technological Educational Institute of Crete, Chania, Crete, Greece
}

Article history

Received October 12, 2017; accepted November 27, 2017.

Subject classification:

Transient Electromagnetic Method; Tsallis statistics; Karstified basin; Fractional diffusion; Multi-scaled hierarchical geological structure

\begin{abstract}
It is being increasingly recognized that geological media are inherently rough with persistent, long-range spatial correlations in physical properties, including electrical conductivity, which spans many decades in length scale. In the present study, the ideas of a multi-scaled geological medium and the anomalous diffusion of electromagnetic eddy currents applied, in Keritis Basin (Western Crete, Greece), a complex geological system surrounded by normal faults and with the majority of formations to be calcareous and karstified. The evidence of a multi-scaled hierarchical structure is presented,based on observed q-exponential distributions of the resistivity, supporting our motivation to introduce fractional diffusion ideas and non-extensive statistical physics to describe the geoelectrical structure of karstified Keritis basin. The essential goal of this paper is to test in a real geological complex formation the Transient Electromagnetic Method (TEM) response in terms of the rough geological medium where the conductivity of the ground has a spatial distribution, which is described by a roughness parameterand to better understand the geoelectrical properties of complex geological structure introducing the ideas of fractional diffusion and non extensive statistical physics.
\end{abstract}

\section{Introduction}

Electromagnetic methods are widely applicable to the solution of environmental and engineering problems, since they are very effective in applications where the geoelectric structure is simple and the geological medium is characterized by a piecewise smooth spatial distribution of electrical conductivity. However, such a description cannot always be justified and recent results suggest that most of the physical properties of geological media, including electrical conductivity, are inherently rough forming a multiscaled structure [Pilkington and Todoeschuck 1993, Painter 1996, Tennekoon et al.
2005, Molz and Hyden 2006, Beskardes et al. 2017].

In Earth sciences the conceptsof fractal geometry and complexity have been introduced to describe patterns in geophysics, seismicity, volcanology, geomorphology and hydrogeology introducing the fractal properties of multiscale heterogeneity [Vallianatos 1996, Turcotte 1997, White et al. 2002, Bahr et al. 2002, Kiyashchenko et al. 2004, Kouli et al. 2007, Vallianatos and Telesca 2012]. Everett and Weiss [2002] showed that observed electromagnetic responses can be represented as fractal signals due to the inherent multi-scaled structure (called roughness) of the geological medium and placed an emphasis on the need for multiscale analysis to develop complex geology models that would permit the recognition of its electromagnetic signature. In Ge et al. [2012, 2015] the complexity of geological medium introduced, leads to electromagnetic fractional diffusion equation to assess the electromagnetic responses due to a multiscale structured subsurface.

Weiss and Everett [2007] found evidence for the fractional diffusion of electromagnetic eddy currents using time domain electromagnetic (TEM) data. They define a roughness parameter, related to the subsurface fracture density, to characterize the complexity of the geoelectric structure. From all the above mentioned works, it is well documented that anomalous diffusion is strongly associated with spatial variations of material properties. Although the electromagnetic response of geological media is a complicated function of grain/fluid interactions, the spatial hierarchy of a geological formation also strongly influences the geometry of electric current pathways. The inherent presence of multiscale network to most of the structures influ- 
ence their conductivity parameters, resulting in anomalous diffusion. At a microscopic level, classical diffusion is generated by the random motion of individual particles/charges and is traditionally described as a stochastic Gaussian process. However, in anomalous diffusion, the mean-square variance of the particle displacement grows faster or slower than that of a Gaussian diffusion process. Thus, the application of fractional electromagnetism is a challenge to describe the effects of geoelectromagnetic induction in geological formations. All the above mentioned studies, support the idea that real subsurface geometries could be viewed as the superposition of geologic rock types spanning multiple length scales. Across all scales, the corresponding electrical conductivities of the spatial-correlated geologic textures in rocks should generate long-range-dependent, or fractal-like electromagnetic responses.

In a more recent work, Weymeret al. [2015] used the electromagnetic method to evaluate short- and long-range correlations in a large scale geological structure demonstrated that electromagnetic responses are governed by long-range dependence effects. To describe the long-range interaction we can consider the use of statistical physics to understand the collective properties of geostructures. Then a natural question arises. What type of statistical physics is appropriate to describe effects where long-range dependence effects are important? An answer to the previous question could be non-extensive statistical physics (NESP), originally introduced by Tsallis [1988] and recently summarized in Tsallis [2009]. The latter is strongly supported by the fact that this type of statistical mechanics is the appropriate methodological tool to describe entities with (multi) fractal distributions of their elements and where long-range dependence are important as in fracturing phenomena [Vallianatos et al. 2012] and in most patterns appeared in Earth sciences. NESP is based on a generalization of the classic Boltzmann-Gibbs entropy and has the main advantage that it considers all-length scale correlations among the elements of a system, leading to a very common in Earth Sciences asymptotic power-law behavior. A lot of work has been recently done in topics concerning earth sciences in terms of non-extensive statistical mechanics. This work varies from pre-seismic electromagnetic emissions [Kalimeri et al. 2008, Potirakis et al. 2012], Geoelectromagnetism and Space Physics [Balasis et al. 2008, 2009, 2016], evolution of seismicity [Telesca 2010, 2011, Ramirez-Rojas and Flores-Marquez 2011, Papadakis et al. 2013, 2014, Vallianatos et al. 2014, Efstathiou et al. 2015], topics concerning laboratory geophysics [Vallianatos et al. 2012, Vallianatos et al.
2013] and other miscellaneous geophysical topics such as fault population distribution [Vallianatos et al. 2011, Vallianatos and Sammonds, 2011, Vilar et al. 2007, Michas et al. 2015, Papadakis et al. 2016], earthquake energy fluctuations [Wang et al. 2015], rockfalls distribution [Vallianatos 2013] and polarity reversals of Earth's geomagnetic field [Vallianatos 2011]. In addition concepts of universality between different extreme events in the Solar-Terrestrial system is given in Balasis et al. [ $2011 \mathrm{a}, \mathrm{b}, \mathrm{c}$ ]. An extended review of the applications of non-extensive statistical mechanics in Earth Sciences is given in Vallianatos et al. [2015] and Vallianatos et al. [2016].

In this work we study the statistical features of Earth's resistivity in a karstic basin as extracted from TEM soundings introducing the idea of electromagnetic fractional diffusion equation to assess the electromagnetic responses due to a multiscale structured subsurface and viewing the statistical properties of resistivity in terms of non-extensive statistical physics. Our results support the idea that long-range correlations that are present in Earth's structure could be extracted in terms of non extensive statistical physics and to described by q-exponential distributions that obtained using first principles. The scope of this work is not to present a physical model of earth's resistivity structure but rather to present a phenomenological approach that describes fundamental properties that we have to take into account in view of complexity theory.

\section{Geological and Tectonic Settings of Keritis basin}

The geology of Crete in the Southern Aegean sea is considered to be very interesting due to the position of the island (in the central fore arc of the Hellenic Subduction Zone) and the complexity of its tectonic structure [Angelier 1976].

The study area is represented by the Keritis Basin which is situated in the northwestern part of Chania Prefecture, at Crete Island in Greece (Figure 1). The hydrological basin of Keritis represents one of the most important basins in the municipality of Chania [Kanta et al. 2009a, 2009b, 2013, Parisi et al. 2013]. The area is drained by the main river of the basin, named Keritis River. In the area, three different morphological zones can be distinguished: the mountainous, the semi-mountainous and the lowland which comprise, respectively, the south, central and north part of the basin. In particular: a) the mountainous zone comprises the northwest karstic sector of the White Mountains, typical karstic areas which represent all the shapes from the fractured carbonate rocks; b) the semi-mountainous zone, the southern part of the area representing the 


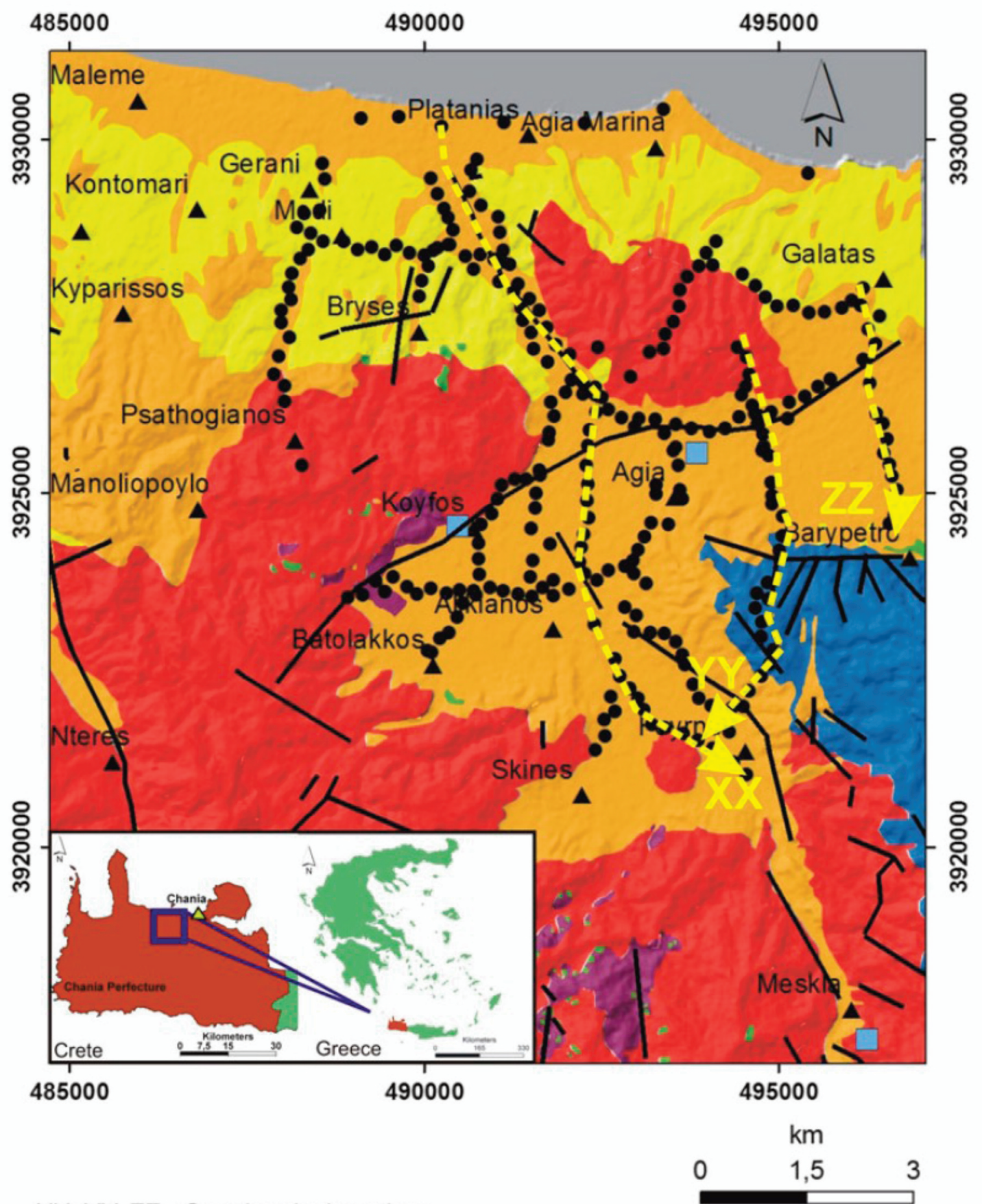

$X X, Y Y, Z Z$ : Geoelectrical sections

$\begin{array}{lll}\text { — Faults } & \text { Quaternary Deposits } & \text { Phyllites-Quartzites } \\ \text { - TDEM Soundings } & \text { Neogene Sediments } & \text { Trypalion Carbonates } \\ \square \text { Springs } & \text { Tripolis Carbonates } & \text { Plattenkalk Limestones }\end{array}$

Figure 1. Geological and tectonic map of Keritis basin (western part of Crete Island, Greece) extracted from Kanta et al. [2009a]. Different colors demonstrate the variety of the geological formations (Quaternary deposits, Neogene sediments, Tripolis carbonates, PhyllitesQuartzites, Trypalion carbonates, Plattenkalk limestone). Solid black lines denote visible/concealed faults. The faults are from the geological maps of IGME (1969) and Lionis and Perleros (2001). The locations of the TEM soundings are also depicted as black solid circles.

transition from the mountainous to the semi-mountainous zone sharply defined by the drop-off of the tectonic structures and c) the lowland zone is the area with soft relief and lowland areas.

The major part of Keritis basin is covered by Quaternary deposits and Miocene sediments that expand to 
the north-east, south-east and south-west. In addition, Pliocene sediments in the north-western part of the study area and the Tripolis nappe also appear in the north-eastern and north-western part of the basin. Dissected hills of Phyllites and Quartzites, a Late Carboniferous to Late Triassic package of sedimentary rocks composed mostly of quartz-rich siliciclastic sediments, with minor limestone, gypsum, and volcanic rocks [Krahl et al. 1983] are observed mainly in the south-western and south-eastern part of the study area.The Trypalion nappe is exposed in the central-eastern part and finally, the Plattenkalk limestone is exposed locally in the south-western part of the study area.

The study area consists of a complex tectonic system surrounded by normal faults. The majority of the formations are calcareous and karstified (high permeability rocks, as that of karstic limestones of Tripolis and Trypalion). The current tectonic regime is dominated by almost north-south and east-west extensions [Lionis and Perleros 2001]. The main strikes of faults are northwest-southeast and east-west (Figure 1), which defines the boundaries between the existing geological and hydrolithological units as well as the groundwater flow direction, as some of the faults bound and direct the groundwater flow [Soupios et al. 2007, Kanta et al. 2009a, Kanta et al. 2013]. The lithologic composition of the aquifer system is described by Soupios et al. [2007] as clayey/silty sand material enhanced with rubbles and gravels, whereas the estimated and measured hydraulic conductivity values of the aquifer are located within the characteristic limits of pure sands and gravels as stated by Soupios et al. [2007]. Figure 1 presents the geological setting of the study area along with the sites where field measurements using Transient Electromagnetic sounding methodology were carried out .

\section{Principles of the central loop Transient electro- magnetic method over a multiscale (rough) geologi- cal medium}

Electromagnetic methods of geophysical exploration are diagnostic of the spatial and, sometimes, the temporal variability in ground electrical resistivity, a macroscopic physical property that is a function of many interconnected parameters including lithology, pore-scale surface chemistry, fracture density, and the presence and distribution of fluids. When an electromagnetic source is applied, the Earth's macroscopic response is a multiscale integration of the effects of charges that build up at conductivity gradients and contrasts and currents that follow specific pathways in accordance with the electrical properties of the medium. The geometry of induced electric current pathways in the subsurface is prescribed by the fundamental electromagnetic laws along with the knowledge of the subsurface electrical conductivity distribution $\sigma(\mathbf{r})$. Overviews of electromagnetic induction are given in a very broad literature [Wait 1951, Nabighiam 1979, Ward and Hohmann 1987, McNeil 1990, Nabighiam and Macnae 1991].

Low-frequency electromagnetic induction in an idealized piecewise smooth Earth is well described by Maxwell equations as a classical diffusion process. In the case of a rough (multi-scaled) geological medium containing long-range-dependent heterogeneities, the importance of the multiscale electrical properties in the electromagnetic responses, only recently has been recognized [Everett and Weiss 2002, Everett 2009]. Multiscale hierarchical geoelectrical structures affect electromagnetic responses in a way that approaches based on coarse, piecewise smooth representations of earth structure, are approximations introducing a lack of understanding of the complexity of a real solid earth structure.

In this paragraph we recapitulate the basic principles of classical TEM method along with an introduction to the anomalous (fractional) diffusion approach used to describe the electromagnetic induction in rough (multiscale) geological structures.

In the central-loop TEM sounding method, a current is induced in a classical diffusion way into the ground by a time-varying magnetic field of a controlled magnitude generated by a source loop. A loop of wire is placed on the ground and a constant magnetic field of known strength is built up by transmitting a constant current into the loop. The current is then abruptly turned off, and the decaying magnetic field induces electromotive forces in the ground. The induced current is initially concentrated below the transmitter loop, but then the current diffuses down and away from the transmitter. The current distribution in the ground generates a secondary magnetic field that decays with time. The decay rate of the secondary magnetic field as a function of time is monitored by measuring the voltage induced in a receiver coil at the centre of the transmitting loop in time gates. The current distribution and the decay rate of the secondary magnetic field depend on the resistivity structure of the earth. The decay rate, recorded as a function of time after the current in the transmitter loop is turned off, can therefore be interpreted in terms of the subsurface resistivity structure [Arnason 1989, Nabighiam and Macnae 1991].

In recent works [Swidinsky and Weiss 2017, Swidinsky and Nabighian 2015] the transient induction $\log$ response in a half space of conductivity $\sigma$ and free 
space magnetic permeability $\mu_{\mathrm{o}}$ have been presented in a compact analytical way. An analytical expression for the azimuthal electric field $\mathrm{E}_{\varphi}$ produced, when a current $I$ is rapidly switched off in a horizontal loop of wire with radius $\alpha$ located at the origin derived as:

$$
E_{\varphi}(r, z, t)=\frac{I \alpha \mu_{o}}{2} \sqrt{\frac{\theta^{2}}{\pi t^{2}}} e^{-\theta^{2}\left(\alpha^{2}+r^{2}+z^{2}\right)} I_{1}\left(2 \theta^{2} \alpha r\right)
$$

where $\theta=\sqrt{\frac{\mu_{\mathrm{o}} \sigma}{4 t}}$ and $\mathrm{I}_{1}$ is a modified Bessel function of the first kind. From Faraday's Law, the voltage induced in a receiver loop coincident with the transmitter wire is:

$$
V(t)=\mu_{o} \pi I \alpha^{2} \sqrt{\frac{\theta}{\pi t^{z}}} e^{-2 \theta^{z} \alpha^{2} I_{1}\left(2 \theta^{2} \alpha^{2}\right)}
$$

In principle one could measure the relaxation time series $V(t)$ and then determine formation's resistivity. However, such a procedure is limited by the non-linear nature of the exponential and Bessel functions. Instead, examination of the late-time response $(t \rightarrow \infty$ and $\theta \rightarrow 0)$ yields a simple expression for the electromagnetic response and the earth's apparent conductivity. The late time-response derived by expanding the Bessel function and the exponential one by a polynomial series in the product $\theta^{2} \alpha^{2}$, yielding [Abramowitz and Stegun 1964]:

$$
I_{1}\left(2 \theta^{2} a^{2}\right)=\theta^{2} a^{2}+\frac{\left(\theta^{z} \alpha^{z}\right)^{z}}{2 !}+\frac{\left(\theta^{z} \alpha^{z}\right)^{z}}{2 ! 3 !}+\ldots
$$

and

$$
e^{-\theta^{z} \alpha^{z}}=1-\theta^{2} \alpha^{2}+\frac{\left(\theta^{z} \alpha^{z}\right)^{z}}{2}
$$

Inserting the expansions into the expression which gives $V(t)$ and keeping the lowest order, which corresponds to late time, we lead to:

$$
V(t) \cong \sqrt{\pi} I \alpha^{4} \mu_{o}\left(\frac{\mu_{o} \sigma}{4}\right)^{3 / 2} t^{-5 / 2}
$$

The aforementioned equation indicated that for a homogeneous half space $V(t)$ scales to $t^{-5 / 2}$ and $\sigma^{3 / 2}$ in the late stage. The response of homogenous half spaces with different resistivities has the same common character and can be divided into three stages: a) in the early stage, where the induced voltage is constant in time, b) in the intermediate stage, where the voltage starts to decrease with time and with steadily increasing slope on log-log scale until the late stage is reached and c) in the third stage, when the voltage response decreases with time in such a way that the logarithm of the induced voltage decreases linearly as a function of the logarithm of time with slope $-5 / 2$ and an induced voltage scaling with conductivity as $\sigma^{3 / 2}$ appears.

For a complex multi-scaled geological structure, the concept of anomalous diffusion must be introduced
[Metzler and Klafter 2000]. As mentioned, fractional diffusion equation for electromagnetic induction originated due to the superposition of responses from individual geological structures that exist at multiple length scales and the mutual induction between them, in order to form the observed electromagnetic response. A classical diffusion equation may be adequate to describe geological media, which can be approximated by blocks of spatially smoothly varying material properties. However, it is the superposition of individual electromagnetic responses from induced currents flowing in layers upon layers of hierarchical structures that combined to form the measured electromagnetic response which is governed by a fractional diffusion equation. Interpretation of electromagnetic geophysical data in terms of a fractal description of subsurface electrical conductivity is not new. Everett and Weiss [2002] showed that profiles of frequency domain controlled-source electromagnetic responses are inherently rough, while Weiss and Everett [2007] derived the electromagnetic fractional diffusion governing equations starting from the conventional Maxwell's equations.

Fractional derivatives naturally arise when one considers transport processes within spatially hierarchical materials [Metzler and Klafter 2000]. A generalized Ohm's law $\mathbf{J}(\mathbf{t})=\sigma^{\star} \mathbf{e}(\mathbf{t})$ has been used in geophysics to model electromagnetic induction in a polarizable medium [Esposito et al. 2017, Mauriello et al. 1996, Patella 2008, Smith et al. 1988]. Typically, modelling EM induction in such a medium is performed in the frequency domain, in which case Ohm's law becomes the product $\mathbf{J}(\omega)=\sigma(\omega) \mathbf{E}(\omega)$, and the governing Maxwell equations are solved for a given frequency assuming a time-harmonic source excitation. In a number of works [Everett 2009, Ge et al. 2012, Ge et al. 2015 and references therein] employed a time-domain convolutional Ohm's law for describing transient electromagnetic induction in a non-polarizable, but spatially rough medium. They incorporate the time-convolution form of Ohm's law within Ampere's law

$$
\nabla \times \mathbf{b}(\mathbf{t})=\mu_{o} \sigma_{\beta} * \mathbf{e}(\mathbf{t})+\mu_{o} \mathbf{J}_{s}(t)
$$

where

$$
\sigma_{\beta} * \mathbf{e}(\mathbf{t})=\int_{0}^{t} \frac{\sigma_{\beta} \mathbf{e}\left(t^{\prime}\right) d t^{\prime}}{\left(t-t^{\prime}\right)^{1-\beta}}
$$

and $\sigma_{\beta}$ is a generalized electrical conductivity with dimensions of $S / \mathrm{m} \mathrm{s}^{\beta}$ and $\mu_{0}$ is the free space magnetic permeability. Accordingly, as proposed by Everett [2009] we can write $\sigma_{\beta} \sim \sigma \tau^{-\beta}$, where $\tau$ is a parameter with dimensions of time. Note that this form for the generalized electrical conductivity $\sigma_{\beta}$ is appropriate for the anomalous diffusion description of a spatially rough 
medium and the quantity $\sigma_{\beta}$ is referred to as the "anomalous electrical conductivity" in Weiss and Everett [2007].

In case where the geological medium is homogeneous, the induction current diffuses in classical terms. In contrast, in real earth we observe variations in spatial heterogeneity and thus an anomalous diffusion of the induction current. The anomalous diffusion can be generated by a random walk within the complex geometry of a disordered system [Hamrouni and Abdenndher 2016, Juhász and Iglói 2010]. The parameter $\beta$ introduced in the definition of anomalous conductivity, express the ability of fractal transport of charge carriers within a complex geometry (disordered medium) whose heterogeneities obey a spatial power law distribution. In this case the step lengths or equivalently the waiting times follow the theory of continuous time random walk (CTRW), and the parameter $\beta$ describes the roughness of the medium and is related with the waiting time distribution of a charge carrier in a fractal geoelectrical network. Thus $\beta$ could be a parameter that describes the degree of fracturing in the geological formation and increasing of $\beta$ is associated with the increase of fracturing in the geological formation. Alternatively, $\beta$ describes the non-Gaussian step-length distribution function in CTRW and a low $\beta$ value indicates shorter step-lengths and thus a smaller fracture density, while high $\beta$ values are associated with longer step-lengths and increasing density of large fractures [Chi et al. 2014, Elliot et al. 2014].

As a consequence of the above the generalized Ohm's law used and the electromagnetic induction becomes an anomalous diffusion process in a geologically roughmulti-scaled medium. It can be shown that under general conditions, the above equations describe anomalous diffusion [Metzler and Klafter 2000, Caputo 2003, Weiss and Everett 2007] and the convolutional diffusion equation for the electric field can be expressed as a fractional diffusion equation where we take into account the generalized Ohm's law as a convolutionexpression given by:

$$
\frac{\vartheta}{\vartheta t}\left(\sigma_{\beta} * \mathbf{e}\right)=\frac{\sigma_{\beta}}{\Gamma(\beta)} \frac{\vartheta}{\vartheta t}\left(\int_{0}^{t} \frac{\mathbf{e}\left(t^{\prime}\right) d t^{\prime}}{\left(t-t^{\prime}\right)^{1-\beta}}\right)=\sigma_{\beta} \mathrm{o} D_{t}^{1-\beta} \boldsymbol{e}(\boldsymbol{t})
$$

where $\Gamma(x)$ is the Gamma function serving as a normalizing constant and the time derivative as expressed by the fractional derivative $\mathbf{D}_{t}^{1-\beta} \boldsymbol{e}(\boldsymbol{t})$ or Riemann-Louiville operator [Caputo 2003 and references therein]. The Riemann-Louiville definition of a fractional order derivative is the fundamental governing operator of a fractional transport process and is a direct result from Continuous Time Random Walk theory [Metzler and
Klafter 2000] emphasizing the crucial role of anomalous diffusion in the electromagnetic induction in a multiscale structure. Combining the fundamental equations presented with Faraday's law, following Everett [2009] we can eliminate the magnetic field and obtain a fractional differential equation for the Electric one given as:

$$
\nabla \times \nabla \times \mathbf{e}(t)=-\mu_{o} \sigma_{\beta}{ }_{o} D_{t}^{1-\beta} \mathbf{e}(\boldsymbol{t})-\mu_{o} \frac{\vartheta \mathbf{J}_{s}}{\vartheta t}
$$

from which we get the fractional diffusion equation

$$
\nabla^{2} \mathbf{e}(t)=\mu_{o} \sigma_{\beta 0} D_{t}^{1-\beta} \boldsymbol{e}(\boldsymbol{t})
$$

for the out of the source area. The fractional diffusion Equation (xx) is conveniently solved in the Laplace's domain since the Laplace transformation of the operator yield to $L\left\{{ }_{0} D_{t}^{1-\beta} \mathbf{e}(\mathrm{t})\right\}=s^{1-\beta} \mathbf{e}(\mathrm{s})$ [Abramowitz and Stegun 1964, Metzler and Klafter 2000], the Laplace transform of the Equation (7a) is

$$
\nabla \times \nabla \times \mathbf{e}(s)=-\mu_{o} \sigma s^{1-\beta} \mathbf{e}(s)-\mu_{o} s \mathbf{j}_{s}(s)
$$

while that of $(7 b)$ results to the fractional diffusion equation

$$
\nabla^{2} \mathbf{e}(s)=\mu_{o} \sigma_{\beta} s^{1-\beta} \mathbf{e}(s)
$$

where $\mathbf{e}(\mathbf{s})$ is denoted as the Laplace transform of the electric field and $s$ is the Laplace variable. It is clear that in the multi-scaled geological structure, Equations (8, a,b) are identical to the classical problem [Nabighiam and Macnae 1991] except that, in the first term on the right-hand side of equations we have $s^{1-\beta}$ instead of $s$. The solution to the rough geology problem generalizes classical solutions (corresponding to the limit $\beta \rightarrow 0$ ) for the electromagnetic response of a loop over a uniform conducting half-space. Worth to mentioned that in the special case of a smooth medium in which the roughness parameter $\beta \rightarrow 0$, the fractional diffusion reduces to the classical diffusion equation $\nabla \times \nabla \times \mathbf{E}=-\mu_{0} \partial\left(\sigma \mathrm{E}+\mathrm{J}_{\mathrm{S}}\right) / \partial \mathrm{t}$ with a scaling law $\mathrm{V} \sim \mathrm{t}^{-5 / 2}$. In Hanstein [2009] the response of a vertical magnetic dipole in frequency domain over a rough conductive media is given as

$$
h_{z}(s)=\frac{M}{2 \pi r^{3}} \frac{1}{u^{2}}\left[-9+\left(9+9 u+4 u^{2}+u^{3}\right) e^{-u}\right]
$$

with the fractional induction number $u=i k_{\beta} r=i\left(s^{1-\beta} \mu_{o} \sigma\right)^{1 / 2} \mathrm{r}$. In the case where $\beta=0$ the expression is identical to that of Ward and Hohmann [1987]. For normal diffusion with roughness equal zero the transient response can be also given in analytical expression but for a general fractional induction number with the roughness pa- 
rameter $\beta$ the transformation to time domain has to be done by numerical techniques. Only the asymptotic limits for the high and low frequency limit can be calculated in closed form. For high frequency and early time we get $h_{z}^{h f}(s)=-\frac{M}{2 \pi r^{2}} \frac{9}{u^{2}}$ and the response in time domain is

$$
H_{Z}^{e r}(t)=-\frac{M}{2 \pi r^{3}} \frac{9}{\mu_{o} \sigma r^{2}} \frac{1}{\Gamma(1-\beta)} t^{-\beta}
$$

For low frequency approximation we can developed a series expansion of the magnetic field as

$$
h_{z}^{l f}(s)=-\frac{M}{2 \pi r^{3}} \frac{1}{2}\left[-1-2\left(\sum_{n=4}^{\infty} \frac{(n-1)(n-3)^{2}}{n !}(-u)^{n-2}\right]\right.
$$

where the first three terms of the expansion are important to approximate the late time behavior given as

$$
h_{z}^{l f}(s) \approx-\frac{M}{2 \pi r^{3}} \frac{1}{2}\left[-1-\frac{1}{2} u^{2}+\frac{4}{15} u^{3}-\ldots\right]
$$

In time domain the first term in the above expression is a $\delta$-function without influence on late time decay while for the second term taking into account the inverse Laplace transform $L^{-1}\left(s^{1-\beta}\right)=-\frac{\Gamma(\beta)}{\beta-1} t^{-(2-\beta)}$ for $\beta>0$ [Hanstein, 2009] we conclude that it is responsible for the late time behavior in a rough medium. The third term describes the classical decay response in a non-fractional medium and fora fractional medium this term decays faster than the previous second term. Thus, the late time TEM response over a rough (multiscaled) earth with conductivity $\sigma$ is scaling with time as

$$
V(t) \sim \frac{d h_{z}^{l r}}{d t}=\frac{M}{2 \pi r^{3}} \frac{\mu_{o} \sigma \Gamma(\beta)}{2(\beta-1)} t^{-(2-\beta)}
$$

with $0<\beta<1$.

The cause for the heavy tailed decay response in rough geological media can be explained by the additive second term in the series expansion which yield to a continuous transition from non-fractional to fractional diffusion. Equation (13) is an expression of the form $V(t)$ $\approx V_{o} t^{-\gamma(\beta)}$ with $\gamma(\beta)=2-\beta<2$ and $V_{o}(\sigma, \beta, M)$ is linearly scaled with conductivity $\sigma$ in contrast to the homogeneous earth case where $V_{0}$ scaled with $\sigma^{3 / 2}$. The transient decay for normal diffusion is $\mathrm{t}^{-5 / 2}$ and for anomalous diffusion the decay is slower.

\section{TEM data analysis in terms of non extensive sta- tistical physics}

A survey was carried out in Keritis basin where $312 \mathrm{TEM}$ soundings were acquired in different locations creating a detailed survey grid (about 200-250 $\mathrm{m}$ in X and Y dimension) using a 50x50 m single loop configuration. The TEM data collected using TEM-Fast 48 system and anal- ysed using the TEM-RES software [Barsukov and Fainberg 2002, Barsukov et al. 2007]. A typical TEM response presented in Figure 2 which in view of Equation (13) corresponds to $\beta \approx 0.2$.Using the approach presented in Svetov and Barsukov [1984] the TEM data inverted to create a resistivity model of the area presented in Kanta et al. [2009b] and Kanta et al. [2013] and a multiscale heterogeneity suggested as a result of the spatial variability of geological processes which operates across multiple length scales. The relationship between the multiscale geoelectrical heterogeneity and the TEM response has not been completely understood due to the limited observations and the insufficiency of traditional modelling approaches which oversimplify the Earth structure as an idealized piecewise smooth medium. To study the existence of any statistical and hierarchical pattern in the resistivity structure, we use the parameter $\mathrm{V}_{\mathrm{o}}(\sigma)$ of Equation (13) which linearly scales with $\sigma=1 / \rho$. Since Equation (13) is the late time TEM response we select the soundings where $\gamma(\beta)<2$. Furthermore, our results are related with layers $\mathrm{E}$ and $\mathrm{F}$ (depth of the order of $100-150 \mathrm{~m}$ ) as presented in Figure 4 of Kanta et al. [2013]. In order to study the spatial multiscaling character of the structure, in a universal formalism we introduce the normalized

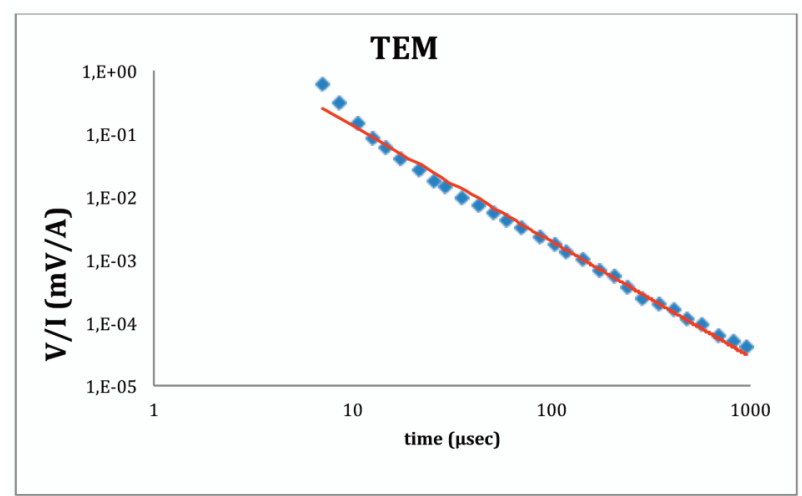

Figure 2. Example of TEM response in Keritis karstic zone, with rough parameter $\beta \approx 0.2$.

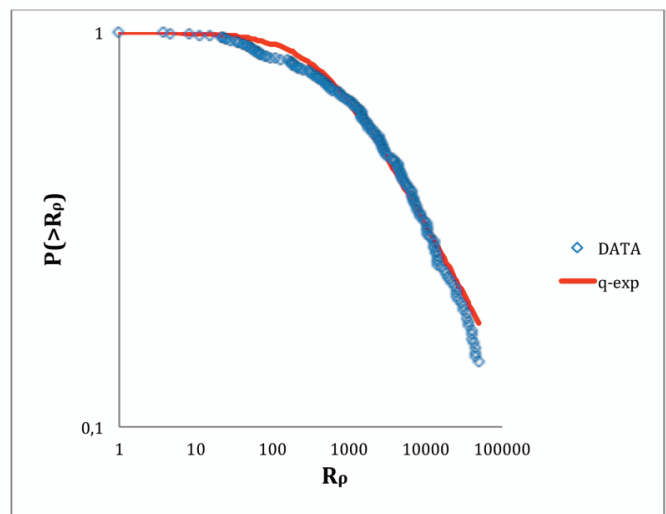

Figure 3. The cumulative distribution of normalized resistivities observed for the Keritiskarstic basin along with the q-exponential fitting with $\mathrm{q}=3.725$ (see text). 
resistivity $R_{\rho}=\frac{\rho}{\rho}=\frac{V_{\text {omax }}}{V}$. We plot the normalized cumulative distribution function of normalized resistivity $\mathrm{P}\left(>\mathrm{R}_{\rho}\right)$ which counts the number of measured points with normalized resistivity greater than $\mathrm{R}_{\rho}$, normalized by the total number of points used, as a function of normalized resistivity $R_{\rho}$. Figure 3 presents the $\mathrm{P}\left(>\mathrm{R}_{\rho}\right)$ versus $\mathrm{R}_{\rho}$ distribution, using $\mathrm{R}_{\rho}$ values extracted for the TEM soundings with $\gamma<2$, for the Keritis karstic basin. It is obvious that in the distributions a power law tail is observed where $\mathrm{P}\left(>\mathrm{R}_{\rho}\right) \sim \mathrm{R}_{\rho}^{-\mathrm{k}}$, with $\kappa \approx 0.35$.

Next step is to interpret the distribution $P\left(>R_{\rho}\right)$ in terms of the Non-Extensive Statistical Physics (NESP) motivated from the fact that NESP has been extensively used to describe anomalous (fractional) diffusion phenomena [Lenzi et al. 2003a, Lenzi et al. 2003b, Prehlet al. 2012, Reynolds and Geritz 2015]. Non-Extensive Statistical Physics introduced by Tsallis [1998, 2009] generalizes Boltzmann-Gibbs statistical mechanics in order to explain the behavior of complex systems where (multi)fractal structuresand long-range correlations are important, leading to power-law scaling. A detailed analysis of the properties, limitations and applications of NESP to Earth sciences can be found in a recent review by Vallianatos et al. [2015, 2016]. One of the most important principles in NESP is the maximization of the Tsallis non-additive entropy [Tsallis 2009], defined as:

$$
S_{q}=k_{B} \frac{1-\sum_{i=1}^{w} p_{i}^{q}}{q-1}
$$

The parameter " $\mathrm{q}$ " in the definition of Tsallis entropy reflects the degree of non-additivity, $\mathrm{W}$ is the total number of microscopic states and pi is a set of probabilities. When $\mathrm{q}=1$ the Boltzmann- Gibbs theory is recovered. In contrast to the B-G entropy, the Tsallis entropy is non-additive. The latter implies that, for two probabilistically independent systems A, B the Tsallis entropy satisfies the following equation:

$$
\frac{S_{q}(A+B)}{k_{B}}=\frac{S_{q}(A)}{k_{B}}+\frac{S_{q}(B)}{k_{B}}+(1-q) \frac{S_{q}(A)}{k_{B}} \frac{S_{q}(B)}{k_{B}}
$$

Non-additivity is defined by the last term on the right-hand side of the equation and reflects superadditivity, additivity and subadditivity when $\mathrm{q}<1, \mathrm{q}=1$ and $\mathrm{q}>1$ respectively, and this is the main principle of NESP. Using the Lagrange multipliers method, we can maximize the non-extensive entropy under appropriate conditions [Tsallis 2009, Vallianatos et al. 2015, 2016], in order to find the probability distribution $p(X)$ of a certain parameter $\mathrm{X}$, which in our study refers to the normalized resistivity $\mathrm{R}_{\rho}$. The probability distribution $\mathrm{p}\left(\mathrm{R}_{\rho}\right)$ after applying the Lagrange multipliers method is given as:

$$
p\left(R_{p}\right)=\frac{\left[1-(1-q)\left(\frac{R_{\rho}}{R_{o \rho}}\right)\right]}{Z_{q}}=\frac{\exp _{q}\left(-\frac{R_{\rho}}{R_{o \rho}}\right)}{Z_{q}}
$$

where $Z_{q}$ is the $q$-partition function $Z_{q}=\int_{0}^{X}{ }_{\max } \exp _{q}(-X / X o) d X$, and $\exp _{q}(X)$ is the q-exponential function that is defined as $\exp _{q}(X)=[1+(1-q) X]^{\frac{1}{1-q}}$ when $[1+(1-q) X] \geq 0$ and zero when $[1+(1-q) X]<0$ while the cumulative distribution is given as

$$
P\left(>R_{p}\right)=\exp _{q}\left(-\frac{R_{\rho}}{R_{o \rho}}\right)
$$

In the limit $\mathrm{q} \rightarrow 1$ the $\mathrm{q}$-exponential functions lead to the ordinary exponential functions. If $\mathrm{q}>1$ asymptotic power-law behavior is observed with slope -1/ (q1) [Abe and Suzuki 2005, Vallianatos et al. 2015].

To test the q-exponential description of the $\mathrm{P}\left(>\mathrm{R}_{\rho}\right)$ versus $\mathrm{R}_{\rho}$ distribution we fit the data with Equation (17). Figure 3 present the original data with the q-exponential Equation (17) with $\mathrm{q}=3.725$, which suggests that the distribution of resistivity in the karstic Keritis basin presents an hierarchical patters which is consistent in statistical terms with that of non-extensive statistical mechanics. We note that equation (17) has an asymptotic behaviour $P\left(>R_{\rho}\right) \sim R_{\rho}^{-1 / q-1}$ leading to a power law with exponent $k=\frac{1}{q-1} \approx 0.367$ in an agreement with
$\kappa \approx 0.35$ observed.

\section{Concluding remarks}

It is being increasingly recognized that geological media are inherently rough with persistent, long-range spatial correlations in physical properties, including electrical conductivity that span many decades in length scale. Traditional electromagnetic modelling utilizes piecewise smooth representations of the geoelectric structure.

In the present study, motivated by previous EM field studies [Everett and Weiss 2002; Weiss and Everett 2007] along with recent advances in fractional diffusion equations [Metzler and Klafter 2000], the analysis in transient electromagnetic response of a loop switched off in order to search the multiscaling characteristics in the karstified Keritis basin (Western Crete), which has been investigated in the past for its conductivity structure and the asscociated hydrogeophysical properties [Kanta et al. 2009b, Kanta et al. 2013] is applied.

We present evidence of a fractured multi-scaled hierarchical structure, since the extracted from the analysis of electromagnetic responses normalized resistivities follow a q-exponential distribution in accordance with non extensive statistical mechanics which is the appropriate frame to describe complex systems where 
(multi)fractal structures and long-range correlations are important, leading to power-law scaling. The later observation supports our motivation to introduce fractional diffusion ideas to describe the Keritis basin.

Summarizing, we can state that Time Domain Electromagnetic induction data presented here indicate that classical diffusion is not always the most compact physically based description when interpreting geophysical interrogations of the subsurface. Our data instead, suggest that a fractional diffusion mechanism based on a simple but powerful physical justification, may apply important role in electromagnetic induction in formations where hierarchical networks can induce distinctive dynamic processes such as anomalous diffusion. The fractional diffusion approach (even if it is not unique) has advantages over the classical approach since presents a more realistic description of the rough structure of geologic materials. The physical parameters as that of resistivity represent the hierarchical networks and follow q-exponentials as expected in terms on Tsallis entropy.

Even the resistivity distribution extracted by an inversion procedure [Svetov and Barsukov 1984] leads to a resistivity distribution that expressed by a power law [Vallianatos et al 2018 in preparation], it is recommended that additional electromagnetic field studies should be carried out in different geological settings to provide support for the theory set forth in this paper and to develop relationships between the apparent roughness parameter $\beta$ and important geological variables such as lithology, porosity, fracture density, and clay and water content.

It is hoped that the concepts presented in this paper will stimulate further research along these lines into innovative approaches for the interpretation of EM data. The essential goal of this paper is to test in a real geological complex medium the TEM response in terms of the rough geological medium and to better understand the physics that is introduced in terms of complexity theory.

\section{References}

Abe, S. and N. Suzuki (2005). Scale-free statistics of time interval between successive earthquakes, Physica A, 350, 588-596.

Abramowitz, M and I.A. Stegun (1964). Handbook of Mathematical Functions with Formulas, Graphs, and Mathematical Tables. National Bureau of Standards Applied Mathematics Series.

Angelier, J. (1976). Sur la neotectonique de l'arcegeen externe: les failles normales Est-Quest et l'extension submeridienne cretoise. C.R. Acad. Sci. Paris, (D),
282, 413-416.

Árnason, K. (1989). Central loop transient electromagnetic sounding over a horizontally layered earth.Orkustofnun, Reykjavík, report OS89032/JHD-06, 129 pp.

Bahr, K. M. Smirnov, E. Steveling and BEAR Working Group, (2002). A gelation analogy of crustal formation derived from fractal conductive structures. Journal of Geophysical Research: Solid Earth , 107, B11: ECV 18-1-ECV 18-10, doi:10.1029/2001JB000506

Balasis, G., I. A. Daglis, C. Papadimitriou, M. Kalimeri, A. Anastasiadis and K. Eftaxias (2008). Dynamical complexity in Dsttime series using non-extensive Tsallisentropy, Geophys. Res. Lett., 35, L14102, doi:10.1029/2008GL034743.

Balasis, G., I. A. Daglis, C. Papadimitriou, M. Kalimeri, A. Anastasiadis and K. Eftaxias (2009). Investigating dynamical complexity in the magnetosphere usingvarious entropy measures, J. Geophys. Res., 114, A00D06, doi:10.1029/2008JA014035.

Balasis, G., I. A. Daglis, C. Papadimitriou, A. Anastasiadis, I. Sandberg and K. Eftaxias (2011a). Quantifying Dynamical Complexity of Magnetic Storms and SolarFlares via NonextensiveTsallis Entropy, Entropy, 13, 1865-1881,doi:10.3390/e13101865.

Balasis, G., C. Papadimitriou, I. A. Daglis, A. Anastasiadis, I. Sandberg and K. Eftaxias (2011b), Similarities between extreme events in the solar-terrestrial systemby means of nonextensivity, Nonlinear Processes in Geophysics, 18, 563-572.

Balasis, G., I. A. Daglis, A. Anastasiadis, C. Papadimitriou, M. Mandea and K.Eftaxias (2011c). Universality in solar flare, magnetic storm and earthquake dynamicsusing Tsallis statistical mechanics, Physica A, doi:10.1016/j.physa.2010.09.029, 390,341-346.

Balasis, G., S. M. Potirakis, and M. Mandea (2016). Investigating DynamicalComplexity of Geomagnetic Jerks using Various Entropy Measures, Front. Earth Sci.,4:71, doi:10.3389/ feart.2016.00071.

Barsukov, P.O.and E. B. Fainberg (2002).Study of the environment by the transient electromagnetic method using the induced polarization and superparamagnetic effects.Izvestiya, Physics of the Solid Earth 38 (11), 981-984.

Barsukov, P.O., E.B. Fainberg and E.O. Khabensky (2007). Shallow Investigations by TEM-FAST Technique: Methodology and examples. In: Electromagnetic Sounding of the Earth's Interior, Spichak, V.V. (Ed.), Holland: Elsevier, 55-77.

Beskardes, G.D., C.J. Weiss and M. E. Everett (2017). Estimating the power-law distribution of Earth electrical conductivity from low-frequency, con- 
trolled-source electromagnetic responses. Geophysical Journal International, 208, 2, 639-651.

Caputo, M. (2003). Diffusion with space memory modelled with distributed order space fractional differential equations. Annals of Geophysics, 46(2), 223-234.

Chi, L., M. Elliott, Z.Heidari and M. E. Everett (2014). Assessment of micro-fracture density using combined interpretation of NMR relaxometry and electromagnetic logs. Society of Petroleum Engineers - SPE/AAPG/SEG Unconventional Resources Technology.

Efstathiou, A. Tzanis and F. Vallianatos. (2015). Evidence of non extensivity in the evolution of seismicity along the San Andreas Fault, California, USA: An approach based on Tsallis statistical physics. Physics and Chemistry of the Earth, 8586:56-68.

Elliott, M., M. E. Everett and Z. Heidari. (2014). Modeling the effects of anomalous electromagnetic diffusion on induction logs: The next step in mapping natural fracture corridors .Proceedings - SPE Annual Technical Conference and Exhibition, 6: 48694881SPE.

Esposito, R., A. Troiano, M. G. Di Giuseppe, D. Patella and R. M. Castelo Branco (2017). The magnetotelluric response over a $3 \mathrm{D}$ polarizable structure. Journal of Geophysics and Engineering, 14(3), 698712 .

Everett, M.E. and C. J. Weiss (2002). Geological noise in near-surface electromagnetic data. Geophys. Res. Lett,29, 2001GL014049

Everett, M.E. (2009). Transient electromagnetic response of a loop source over a rough geological medium, Geophysical Journal International, 177 (2), 421-429.doi: 10.1111/j.1365-246X.2008.04011.x

Ge, J., M. E. Everett and C.J. Weiss (2012). Fractional diffusion analysis of the electromagnetic field in fractured media Part I: 2D approach.Geophysics, 77(4),WB213WB218.

Ge, J., M.E. Everett, and C.J. Weiss(2015). Fractional diffusion analysis of the electromagnetic field in fractured media-Part 2: 3D approach, Geophysics, 80(3), E175E185.

Hamrouni, W. and A. Abdennadher (2016). Random walk's models for fractional diffusion equation. Discrete and Continuous Dynamical Systems - Series B 21(8), 2509-2530.

Hanstein, T. (2009). TEM with anomalous diffusion in fractal conductive media, Proceedings of the 23rdSchmucker-Weidelt-Colloquium for Electromagnetic Depth Research, editors: Oliver Ritter and
Ute Weckmann ISSN 2190-7021.

Juhász, R. and F. Iglói (2010). Anomalous diffusion in disordered multi-channel systems, Journal of Statistical Mechanics: Theory and Experiment-2010 (3), P03012.

Kalimeri, M., C. Papadimitriou, G. Balasis and K. Eftaxias (2008). Dynamical complexity detection in pre-seismic emissions using non additive Tsallisentropy. Physica A: Statistical Mechanics and its Applications, 387(5-6): 1161-1172.

Kanta, A., P. Soupios, F. Vallianatos and D. Rust (2009a). Geophysical characterization and delineation of the Keritis Basin, Crete Island, Greece using the time domain electromagnetic method (TDEM), 3rd AMIREG International Conference: Assessing the Footprint of Resource Utilization and Hazardous Waste Management, Athens, Greece.

Kanta, A., P. Soupios, F. Vallianatos, D. Rust and P. Barsukov (2009b). The Application of Time Domain Electromagnetic Method to Characterize the Keritis Basin, in Western Crete, Greece, 3rd IASME International Conference on Geology and Seismology, Cambridge, UK, 2009.

Kanta, A., P. Soupios, P. Barsukov, M. Kouli and F. Vallianatos (2013). Aquifer characterization using shallow geophysics in the Keritis Basin of Western Crete, Greece. Environmental Earth Sciences 70(5), 2153-2165.

Kiyashchenko, D., N. Smirnova, V. Troyan, E. Saenger and, F. Vallianatos (2004). Seismic hazard precursory evolution: fractal and multifractal aspects, Physics and Chemistry of the Earth, 29, 367-378.

Kouli, M., F. Vallianatos, P. Soupios and D. Alexakis (2007). GIS-based morphometric analysis of Keritis and Tavronitis drainage networks, Western Crete, Greece, Journal of Environmental Hydrology, 15, 1-17.

Krahl J, G. Kauffmann, H. Kozur, D. Richter, O. Forster and F. Heinritzi (1983). Neue Daten zur Biostratigraphie und zur tektonischen Lagerung der PhyllitGruppe und der Trypali-GruppeaufderInselKreta (Griechenland). GeolRundsch 72:1147-1166.

Lenzi, E.K., R. S. Mendes, K. S. Fa, L. C. Malacarne and L. R. Da Silva (2003). Anomalous diffusion: Fractional Fokker-Planck equation and its solutions, Journal of Mathematical Physics, 44(5), 21792185.

Lenzi, E.K., L. C. Malacarne,R. S. Mendes and I. T. Pedron (2003). Anomalous diffusion, nonlinear fractional Fokker-Planck equation and solutions, Physica A: Statistical Mechanics and its Applica- 
tions 319, 245-252.

Lionis M. and B. Perleros (2001). Hydrogeological study in the plain of Chania. Ministry of Agricultural Policy, Department of Hydrogeology and Modeling, Athens (in Greek).

Mauriello, P., D. Patella and A. Siniscalchi (1996). The magnetotelluric response over 2D media with resistivity frequency dispersion. Geophysical Prospecting, 44(5), 789-818 doi: $10.1111 /$ j.13652478.1996.tb00174.x.

McNeil, J.D. (1990). Use of electromagnetic methods for groundwater studies. Geotechnical and Environmental Geophysics, 1(5): 191-218.

Metzler, R. and J. Klafter (2000). The random walk's guide to anomalous diffusion: a fractional dynamics approach, Phys. Rep., 339, 1-77.

Michas, G., F. Vallianatos and P. Sammonds (2015). Statistical Mechanics and scaling of fault population with increasing strain in the Corinth Rift, Earth and Planetary Science letters, 431, 150-163.

Molz, F.J. and P. D. Hyden (2006). A new type of stochastic fractal for application in subsurface hydrology, Geoderma,134: 274-283.

Nabighian, M.N. (1979). Quasi-static transient response of a conducting half-space: an approximate representation, Geophysics,44, 1700-1705.

Nabighian, M.N. and J.C. Macnae (1991).Time domain electromagnetic methods. Electromagnetic methods in applied geophysics 2: Applications, chapter 6, Nabighian, M.N. (Ed.), SEG Publ., 427520.

Painter, S.L. (1996). Evidence for non-Gaussian scaling behavior in heterogeneous sedimentary formations, Water Resour. Res.,32, 1183-1195.

Papadakis, G., F. Vallianatos and Sammonds, P. (2013).Evidence of NonextensiveStatistical Physics behavior of the Hellenic Subduction Zone seismicity. Tectonophysics, 608:1037-1048.

Papadakis, G., F. Vallianatos and P. Sammonds (2014). A Nonextensive Statistical Physics Analysis of the 1995 Kobe, Japan Earthquake.Pure and Applied Geophysics, 172:1923-1931.

Papadakis, G., F. Vallianatos and P. Sammonds (2016). Non-extensive statistical physics applied to heat flow and the earthquake frequency-magnitude distribution in Greece,Physica A: Statistical Mechanics and its Applications, 456: 135-144.

Parisi, S., S. Pascale, F. Sdao, P. Soupios (2013). Assessment and mapping of the intrinsic vulnerability to pollution: An example from Keritis River Basin (Northwestern Crete, Greece). Environmental Earth Sciences 70(6), 2659-2670.
Patella, D. (2008). Modeling electrical dispersion phenomena in Earth materials.Annals of Geophysics, 51(1),159-165.

Pilkington, M. and J. P. Todoeschuck (1993).Fractal magnetization of continental crust.Geophys. Res. Lett., 20, 627-630.

Potirakis, S. M., G. Minadakis and K. Eftaxias. (2012). Analysis of electromagnetic pre-seismic emissions using Fisher information and Tsallis entropy. Physica A: Statistical Mechanics and its Applications, 391(1-2):300-306.

Prehl, J., C. Essex and K. H. Hoffmann (2012). Tsallis relative entropy and anomalous diffusion, Entropy, 14(4), 701-716.

Ramirez-Rojas, A. and E. L. Flores-Marquez. (2011). Non-extensivity analysis of seismicity occurred within four subduction regions in Mexico. Geophysical Research Abstracts, 13(3):5242.

Reynolds, A.M., S.A. H. Geritz (2015). Tsallis distributions, Lévy walks and correlated-type anomalous diffusion result from state-dependent diffusion. Physica A: Statistical Mechanics and its Applications 424, 317-321.

Smith, R.S., P.W. Walker, B. D. Polzer and G. F. West (1988). The time-domain electromagnetic response of polarizable bodies: an approximate convolution algorithm. Geophys.Prospect., 36, 772-785.

Soupios P., M. Kouli, F. Vallianatos, A. Vafidis and G. Stavroulakis (2007). Estimation of aquifer hydraulic parameters from surficial geophysical methods: a case study of Keritis Basin in Chania (Crete-Greece). J Hydrol 338(1-2), 122-131.

Svetov, B.S. and P.O. Barsukov (1984).Transformation of Quasistationary Transient Geoelectrical Processes into Equivalent Wave Processes. Izv.Akad.Nauk SSSR, Fiz.Zemli, 8, 29-37.

Swidinsky, A. and M. N. Nabighian(2015). On smoke rings produced by a loop buried in a conductive halfspace. Geophysics, 80(4), E225-E236.

Swidinsky, A. and C. Weiss (2017). On coincident loop transient electromagnetic induction logging.Geophysics, 82 , 4 , E211-E220 , doi:10.1190/geo20170134.1.

Telesca, L. (2010). Analysis of Italian seismicity by using a nonextensive approach. Tectonophysics, 494(1-2): 155-162.

Telesca, L. (2011). Tsallis-based nonextensive analysis of the southern California seismicity. Entropy, 13(7): 1267-1280.

Tennekoon, L., M. C. Boufadel and J. E. Nyquist (2005). Multifractal characterization of airborne geophysical data at the Oak Ridge facility.Stoch. Environ. 
Res. Risk Assess, 19: 227-239.

Tsallis, C., (1988). Possible generalization of Boltzmann-Gibbs statistics, J. Stat. Phys., 52, 479-487, doi:10.1007 / BF01016429.

Tsallis, C. (2009). Introduction to nonextensive statistical mechanics-Approaching a Complex World, Springer, New York, 1-382.

Turcotte, D.L. (1997). Fractals and Chaos in Geology and Geophysics. Cambridge Univ. Press

Vallianatos, F. (1996). Magnetotelluric response of a random layered Earth, Geophysical Journal International, 125, 557-583.

Vallianatos, F. (2011). A non-extensive statistical physics approach to the polarity reversals of the geomagnetic field. Physica A: Statistical Mechanics and its Applications, 390(10):1773-1778.

Vallianatos, F. and P. Sammonds. (2011). A non-extensive statistics of the fault-population at the Valles Marineris extensional province, Mars. Tectonophysics, 509(1-2):50-54.

Vallianatos, F., E. Kokinou and P. Sammonds (2011). Non Extensive statistical physics approach to fault population distribution. A case study from the Southern Hellenic Arc (Central Crete).Acta Geophysica59(4), 770-784.

Vallianatos, F., P. Benson, P. Meredith and P. Sammonds (2012). Experimental evidence of a non-extensive statistical physics behavior of fracture in triaxially deformed Etna basalt using acoustic emissions, Eur. Phys. Let. (EPL), 97: 58002.

Vallianatos, F. and L. Telesca (2012). Statistical mechanics in earth physics and natural hazards, ActaGeophysica, 60, 3, 499-501.

Vallianatos, F. (2013). On the statistical physics of rockfalls: A non-extensive view. Eur. Phys. Let., (EPL) 101 10007.

Vallianatos, F., G. Michas, P. Benson and P. Sammonds (2013). Natural time analysis of critical phenomena: The case of acoustic emissions in triaxially deformed Etna basalt. Physica A: Statistical Mechanics and its Applications, 392(20), 5172-5178.

Vallianatos F., V. Karakostas and E. Papadimitriou (2014). A Non-Extensive Statistical Physics View in the Spatiotemporal Properties of the 2003 (Mw6.2) Lefkada, Ionian Island Greece, Aftershock Sequence, Pure and Applied Geophysics, 171, 7, 1343-1534, 10.1007/s00024-013-0706-6.

Vallianatos, F., G. Michas and G. Papadakis (2015). A description of seismicity based on Non Extensive Statistical Physics: A review, in: D’Amigo (Ed.), Earthquakes Their Impact Soc., Springer Natural Hazards, Berlin, 1-41. doi:10.1007/978-3-319-21753-6
Vallianatos, F., G. Papadakis and G. Michas (2016). Generalized statistical mechanics approaches to earthquakes and tectonics, Proc. R. Soc. A Math. Phys. Eng. Sci. 472 , 20160497. doi:10.1098/rspa.2016.0497.

Vallianatos, F., M. Kouli and D. Kalisperi (2018). Transient Electromagnetic Method in the complex geological structure of Geropotamos (Crete, Greece): Evidence of hierarchy in a fractured system (submitted).

Vilar, C. S., G. S. Franca, R. Silva and J. S. Alcaniz (2007) Nonextensivity in geological faults? Physica A: Statistical Mechanics and its Applications, 377(1):285290.

Wait, J. R. (1951). The magnetic dipole over the horizontally stratified earth, Can. J. Phys., 29,577-592.

Wang, P., Z. Chang, H. Wang and H. Lu (2015). Scaleinvariant structure of earthquake energy fluctuations for different faulting styles. The European Physical Journal B, 88(8):206.

Ward, S.H. and G.W. Hohmann (1987). Electromagnetic theory for geophysical applications, in Electromagnetic Methods in Applied Geophysics, Vol. 1, 131-311, ed.Nabighian, M.N., SEG Press, Tulsa.

Weiss, C.J. and M.E. Everett (2007). Anomalous diffusion of electromagnetic eddy currents in geological formations. Journal of Geophysical Research: Solid Earth, 112(8): B08102. doi: 10.1029/2006JB004475

Weymer, B.A., M. E. Everett, T. S. de Smet and C. Houser (2015). Review of electromagnetic induction for mapping barrier island framework geology. Sedimentary Geology, 321, 11-24.

White, B.S., W. Kohlerand and L.J. Srnka (2002). Random scattering and the detection capability of the magnetotelluric method. IUTAM Symposium on Mechanical and Electromagnetic Waves in Structured Media Solid Mechanics and Its Applications, 91314, doi:10.1007/0-306-46955-3_3.

\footnotetext{
*Corresponding author: Filippos Vallianatos,

Laboratory of Geophysics and Seismology, UNESCO Chair on Solid Earth Physics and Geohazards Risk Reduction, Technological Educational Institute of Crete, Chania, Crete, Greece; email: fvallian@chania.teicrete.gr
}

C 2017 by the Istituto Nazionale di Geofisica e Vulcanologia. All rights reserved. 